\begin{tabular}{|c|l|}
\hline Title & Structure of large scale flows and their oscillation in the thermal convection of liquid gallium \\
\hline Author(s) & $\begin{array}{l}\text { Y anagisawa, Takatoshi; Y amagishi, Y asuko; Hamano, Y ozo; Tasaka, Y Yji; Y oshida, Masataka; Y ano, Kanako; Takeda, } \\
\text { Y asushi }\end{array}$ \\
\hline Citation & $\begin{array}{l}\text { Physical Review E, 82(1),016320 } \\
\text { https://doi.org/10.1103/PhysRevE.82.016320 }\end{array}$ \\
\hline Issue Date & 2010-07 \\
\hline Doc URL & http://hdl.handle.net/2115/43769 \\
\hline Rights & ○2010 The A merican Physical Society \\
\hline Type & article \\
\hline File Information & PRE82-1_016320.pdf \\
\hline
\end{tabular}

Instructions for use 


\title{
Structure of large-scale flows and their oscillation in the thermal convection of liquid gallium
}

\author{
Takatoshi Yanagisawa, ${ }^{*}$ Yasuko Yamagishi, and Yozo Hamano \\ Institute for Research on Earth Evolution, Japan Agency for Marine-Earth Science and Technology, Yokosuka, Japan \\ Yuji Tasaka, Masataka Yoshida, Kanako Yano, and Yasushi Takeda \\ Graduate School of Engineering, Hokkaido University, Sapporo, Japan
}

(Received 18 January 2010; published 30 July 2010)

\begin{abstract}
This investigation observed large-scale flows in liquid gallium and the oscillation with Rayleigh-Bénard convection. An ultrasonic velocity profiling method was used to visualize the spatiotemporal flow pattern of the liquid gallium in a horizontally long rectangular vessel. Measuring the horizontal component of the flow velocity at several lines, an organized roll-like structure with four cells was observed in the $1 \times 10^{4}-2 \times 10^{5}$ range of Rayleigh numbers, and the rolls show clear oscillatory behavior. The long-term fluctuations in temperature observed in point measurements correspond to the oscillations of the organized roll structure. This flow structure can be interpreted as the continuous development of the oscillatory instability of twodimensional roll convection that is theoretically investigated around the critical Rayleigh number. Both the velocity of the large-scale flows and the frequency of the oscillation increase proportional to the square root of the Rayleigh number. This indicates that the oscillation is closely related to the circulation of large-scale flow.
\end{abstract}

DOI: 10.1103/PhysRevE.82.016320

PACS number(s): 47.20.Bp, 47.27.De

\section{INTRODUCTION}

Thermal convection driven by a vertical temperature gradient, Rayleigh-Bénard convection, is an important problem in fluid dynamics, and it is also the basic configuration for flow patterns and heat transport in engineering and geoscience. A Rayleigh-Bénard convection system is described by the Rayleigh number $\left(\mathrm{Ra}=\alpha g \Delta T L^{3} / \kappa \nu\right)$ and the Prandtl number $(\operatorname{Pr}=\nu / \kappa)$, where $\alpha$ is the volume coefficient of thermal expansion, $g$ is the gravitational acceleration, $\Delta T$ is the vertical temperature difference between the fluid layer, $L$ is the layer thickness, $\kappa$ is the thermal diffusivity, and $\nu$ is the kinematic viscosity. The phase diagram for the convection pattern is represented in a $\mathrm{Ra}$ vs $\mathrm{Pr}$ graph. The pattern changes from steady to turbulent state via a time-dependent state with increases in $\mathrm{Ra}$, and the organized structure of the turbulence, termed a "large-scale flow" with periodic oscillation, is observed in the higher $\mathrm{Ra}$ region for $\operatorname{Pr} \sim 1$ [1,2]. The structure and oscillation of the large-scale flow, with its formation mechanism has been much investigated [3] (recent review). There are studies on large-scale flows with both water $(\operatorname{Pr} \sim 7)$ and gases $(\operatorname{Pr} \sim 1)$. However the aspect ratio of the vessels in these studies is not large, and the flow patterns are strongly constrained by the sidewalls. In lower Pr fluids, the two-dimensional steady roll structure at the onset of convective flow easily becomes time dependent just above the critical $\mathrm{Ra}$, and oscillatory instability such as "traveling-wave convection" in the direction of the roll axis is proposed by the theory [4-6]. Transition to turbulence with increases in $\mathrm{Ra}$ in low-Pr fluids occurs at much lower $\mathrm{Ra}$ than water or gases, and large-scale flow is also expected to emerge easily. However, the way to turbulence and the structure of the large-scale flow in low-Pr convection are not fully understood, mainly because the data of convection pat-

\footnotetext{
*yanagi@jamstec.go.jp
}

terns and flow velocities so far for low-Pr fluid is very limited. Liquid metals are used for the study of the low-Pr region $\left(\operatorname{Pr} \sim 10^{-2}\right)$. These are opaque fluids, so usual optical methods for flow measurements cannot be applied to observe the internal flows. Most experimental studies on liquid metal convection have focused on the measurement of the heat transport of the system (Nusselt number), and the temperature fluctuations at different points in the fluid layer related to turbulence. Horanyi et al. [7] studied liquid sodium convection in a cylindrical cell with a large aspect ratio, and reported a $\mathrm{Ra}$ dependence of the Nusselt number up to $\mathrm{Ra}$ $\sim 10^{6}$. Burr and Muller [8] used a rectangular vessel of large aspect ratio with a sodium potassium alloy, and studied the effect of magnetic field on the flow for $10^{3} \leq \mathrm{Ra} \leq 10^{5}$. Aurnou and Olson [9] used a rectangular vessel of large aspect ratio with liquid gallium, and studied the effect of both magnetic field and rotation for $10^{3} \leq \mathrm{Ra} \leq 10^{4}$. For the extremely turbulent flow region at higher $\mathrm{Ra}\left(10^{6} \leq \mathrm{Ra} \leq 10^{10}\right)$ values, tall cylindrical cells with small aspect ratios have been used to investigate the convection of mercury [10-12]. In these studies, the temperature is monitored in the liquid metal and the existence of large-scale flows is suggested by the temperature fluctuations, however direct measurements of flow velocities have not been realized. Simultaneous measurements of velocity profiles could enable a better understanding of the emergence and characteristics of large-scale flows in low-Pr fluids. By using the ultrasonic velocity profiler (UVP) method [13] Mashiko et al. [14] and Tsuji et al. [15] realized direct measurement of convective velocity profiles for $10^{6} \leq \mathrm{Ra} \leq 10^{11}$ in mercury, and several modes of largescale flows with complex fluctuations have been reported. However, the apparatus here is also a tall cylinder where the flow pattern is strongly constrained by the sidewalls. As a result, the basic structure of large-scale flow in liquid metals and its relation to the oscillatory convection at low $\mathrm{Ra}$ are not fully elucidated, and to achieve them, it is necessary to investigate the region of moderate $\left(10^{4} \leq \mathrm{Ra} \leq 10^{6}\right) \mathrm{Ra}$ values with large aspect ratio cells. 

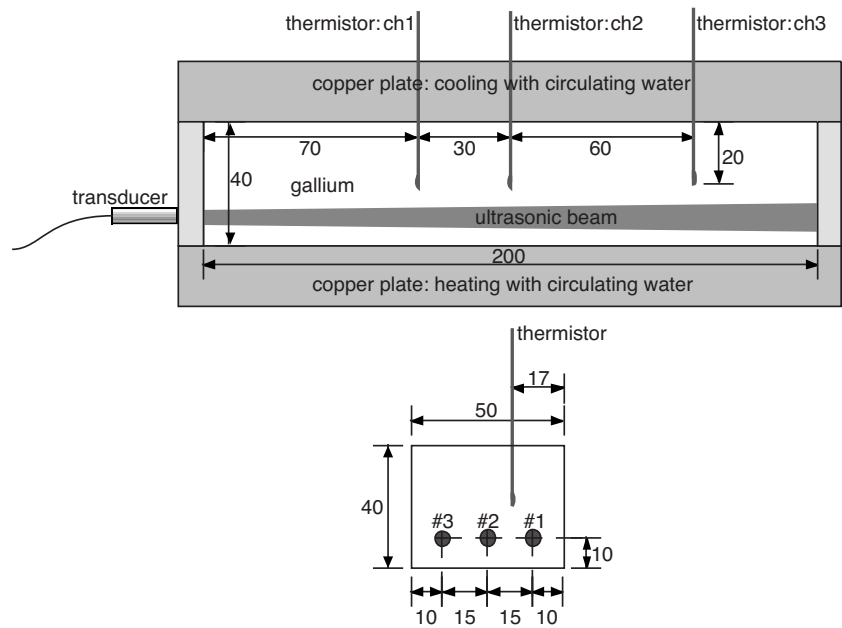

FIG. 1. Side view of the vessel used in the experiments, numbers show the lengths in $\mathrm{mm}$, with the positions of the ultrasonic transducers $(\# 1 \sim \# 3)$ and the thermistor probes $(\mathrm{ch} 1 \sim \operatorname{ch} 3)$ indicated.

The aim of this study is to achieve direct measurements of the velocity profile for Rayleigh-Bénard convection in liquid metals and to visualize the characteristic flow pattern at moderate $\mathrm{Ra}\left(1 \times 10^{4}-2 \times 10^{5}\right)$, which is from about five times to the value where the transition to "oscillatory instability" for $\operatorname{Pr}=0.025$ occurs $(\mathrm{Ra} \sim 2000)[5]$ and above the value for "inertial convection" ( $\mathrm{Ra} \sim 7000)$ [16], to the value of developed turbulence. The UVP method is used for flow velocity measurements. The vessel for the present experiments is horizontally long with an aspect ratio of 5. Tasaka et al. [17] used a similar setup and have shown the existence of celllike flow structures with repeated expansions and contractions in liquid metal convection. This paper further investigates details of the flow structure, and establishes the relation between the flow pattern and temperature fluctuations by simultaneous measurements of the velocity profile and temperature. We also report the $\mathrm{Ra}$ dependence of the typical flow velocities of the large-scale flows and their frequencies of the fluctuations.

\section{APPARATUS AND METHOD}

Figure 1 shows the vessel for the liquid metal convection and the positions of the various measurements points reported in this study. Liquid gallium is used as the working fluid, and the sidewalls of the vessel are $10 \mathrm{~mm}$ thick glass, the glass wetted well with the liquid gallium. The top and bottom plates are made of copper, and the temperature of each plate is maintained constant by circulating water. The inside measurements of the vessel are $200 \mathrm{~mm}$ long, $50 \mathrm{~mm}$ side, and $40 \mathrm{~mm}$ high, giving an aspect ratio of 5 (length to height). The UVP was used to measure the velocity field of the flow in the liquid metal. See [13] for the principles of the method and [17] for details of the experimental procedure with liquid gallium. The transducer for the ultrasonic measurements is attached on the outside of the sidewall, and the ultrasonic waves pass through the glass. The lines of mea- surement for the flow velocity were set along the long horizontal direction of the vessel, and the horizontal component of the flow velocity in this direction at each line was measured. The equipment for the velocity measurement is a UVP-Duo (Met-Flow S. A.), and the basic frequency of the transducer is $4 \mathrm{MHz}$. The diameter of the ultrasonic beam emitted from this transducer is about $5 \mathrm{~mm}$ and it expands to about $10 \mathrm{~mm}$ at the opposite sidewall as shown in Fig. 1. The position of the transducer is selected from the three fixed support positions indicated by \#1 to \#3. We set one or two measurement lines in a series of experiments. The spatial resolution of the velocity measurement determined by the wavelength of the ultrasonic waves in the liquid gallium is $1.43 \mathrm{~mm}$, and the velocity was measured simultaneously at 140 points along the $200 \mathrm{~mm}$ horizontal length of the vessel. The measured value at each point corresponds to the averaged flow velocity of the volume formed by the $1.43 \mathrm{~mm}$ wide slice of the gallium in the vessel. The range of horizontal flow velocities in the present experiments was approximately $\pm 10 \mathrm{~mm} / \mathrm{s}$, and the set value of the velocity resolution was $0.34 \mathrm{~mm} / \mathrm{s}$. The maximum sampling rate for the velocity measurements was $2 \mathrm{~Hz}$, and $\mathrm{ZrB}_{2}$ powder was used as tracer particles because it is approximately neutrally buoyant in liquid gallium. The average diameter of the particles is $50 \mu \mathrm{m}$. There is a small density difference between the tracer particles and liquid gallium $(\sim 1 \%)$, and the particles are gradually sinking even in convecting gallium. Therefore, the reflected signals become weak as the experiments progress, and it is difficult to continue UVP measurements for more than a couple of hours. The melting temperature of gallium is $29.8{ }^{\circ} \mathrm{C}$, the room temperature and the temperature of the circulating water for cooling the top plate was maintained at $32.0{ }^{\circ} \mathrm{C}$, and the temperature of the circulating water for heating the bottom plate was raised to vary the Rayleigh number values, $\mathrm{Ra}$. The outside of the vessel is covered by a thermal insulator to reduce the direct cooling of the walls by the room temperature. The actual vertical temperature differences for the gallium layer were monitored by thermistor probes imbedded in the top and bottom copper plates. The calculations of the $\mathrm{Ra}$ values used the material properties of gallium listed in [18], the Prandtl number of gallium around this temperature is 0.025 . The temperature fluctuations in the gallium layer were measured at three points (ch1, ch2, and ch3 in Fig. 1). The equipment for the temperature measurements is a DS101 with thermistor probes SXK-67 (Takara Thermisor inc.). The size of the thermistor probe head is 1 $\mathrm{mm}$, with a resolution of $0.001 \mathrm{~K}$ and an accuracy of $0.01 \mathrm{~K}$, the sampling rate was $2 \mathrm{~Hz}$.

\section{RESULTS AND DISCUSSION}

\section{A. Structure of the large-scale flow and its fluctuations}

A previous report [17] has used a similar $50 \mathrm{~mm}$ high vessel (aspect ratio 4), and visualized the flow pattern in the gallium layer by measurements of the velocity profile for $10^{5} \leq \mathrm{Ra} \leq 10^{6}$. The horizontal component of the flow velocity in the direction of the ultrasonic beam was measured with a horizontal UVP line, and the results established the organization of the flow structure and its fluctuations. By chang- 


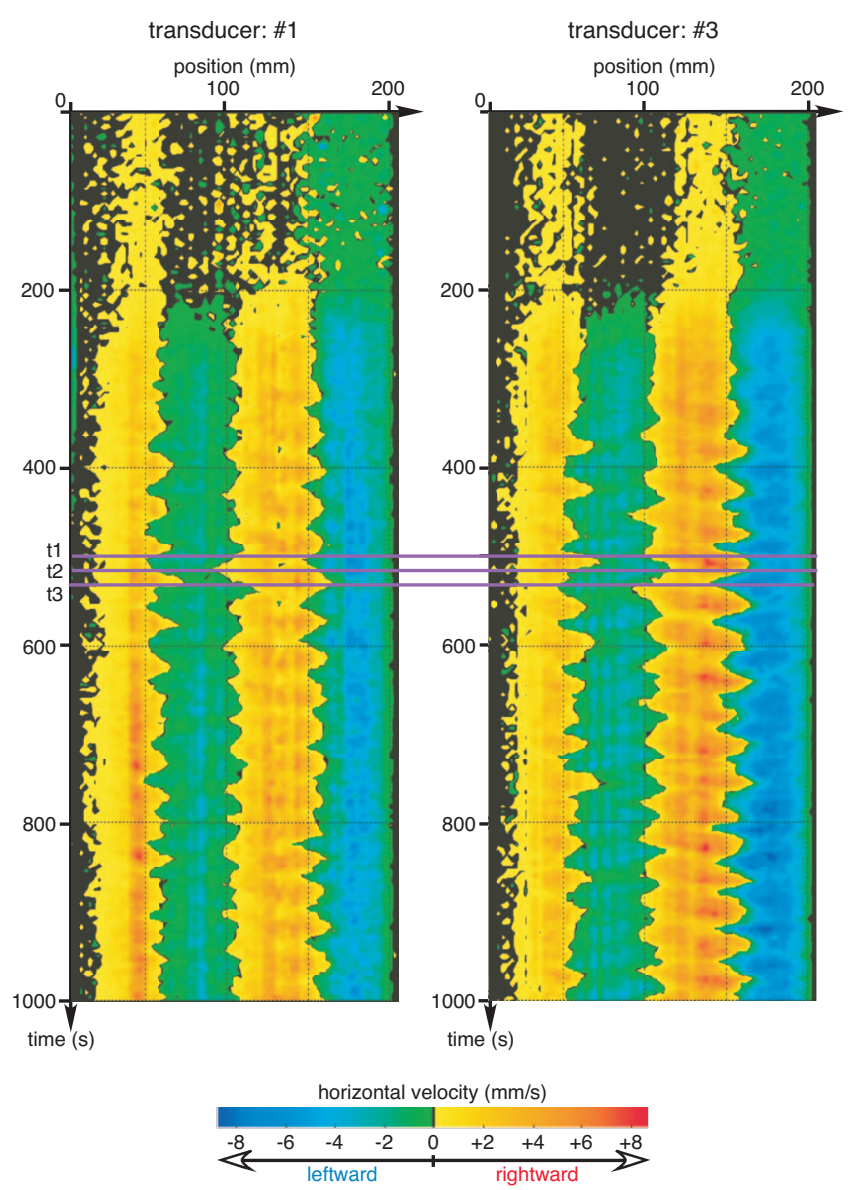

FIG. 2. (Color) The time series of the horizontal flow velocity along two lines of measurement. The positions of transducers \#1 and \#3 are as shown in Fig. 1. The horizontal axis is the position, and vertical axis is the time. The direction and magnitude of the horizontal velocity is displayed in color-scale. The onset of convection occurs after the start of heating of the bottom plate at $100 \mathrm{~s}$, and four clusters of flow are developed after 200 s. The system attains a thermally balanced state after $400 \mathrm{~s}$, and $\mathrm{Ra}=7.8 \times 10^{4}$. The result clearly shows the presence of an organized roll-like flow structure with oscillations.

ing the positions of the line of measurement, the basic flow structure was interpreted as quasi-two-dimensional roll-like cells with the axis of rotation perpendicular to the long horizontal length of the vessel. This result was supported by the fact that the opposite flow direction was observed in the upper and lower parts of the layer and the same direction was observed at horizontally parallel positions. The measurements used only one transducer at a time, and the data set was not sufficient to infer the time variations in the threedimensional flow structure. This section reports the threedimensional structure of the organized flow established in the present investigation by measuring the velocity along two lines with short time lags. Figure 2 shows the velocity measurements by the UVP for the liquid gallium. This experiment indicates the developing process of convective flow. The positions of the ultrasonic transducers are \#1 and \#3 in Fig. 1. The active transducer is alternated every $0.5 \mathrm{~s}$. The horizontal axis is the horizontal distance from the transducer, and the vertical axis is the time elapsed from starting the velocity measurements, the figure shows measurements for 1000 s. The flow velocity parallel to the direction of the measurement line is displayed in color scale: yellow to red indicates the velocity moving away from the transducer (rightward in the figure), and green to blue is the velocity moving toward the transducer (leftward in the figure). The range of the measured horizontal velocity is $\pm 6 \mathrm{~mm} / \mathrm{s}$ in this case. From 0 to $100 \mathrm{~s}$, the temperature of the bottom plate is the same as that of the top plate, and the system is in an almost isothermal state. At $100 \mathrm{~s}$, the temperature of the bottom circulating water was raised. A balanced temperature state is established about $400 \mathrm{~s}$ with a $3.7 \mathrm{~K}$ temperature difference between the top and bottom plates, with the corresponding Rayleigh number $7.8 \times 10^{4}$. The convective motion is turbulent with this $\mathrm{Ra}$ as shown by temperature measurements in the later section and a wide range of length scale might exist, but it is not possible to determine smallscale structures with the UVP measurements because of the low velocity of the small-scale motion.

The dominant feature in Fig. 2 is the emergence of a coherent structure. From 0 to 200 s, the flow velocity is below or comparable to the resolution of the UVP measurements and no distinct structural characteristics are observed. From $200 \mathrm{~s}$, there are four clusters of the positive and negative velocities, indicating the existence of four convection cells in the vessel as the organized flow structure. These cells have a two-dimensional roll-like structure with the axes of rotation perpendicular to the line of the velocity measurements. We term individual, distinguishable cells as 'roll' hereafter. Because the lines of measurement are in the lower half of the gallium layer, the converging boundary corresponds to the upwelling region and the diverging boundary corresponds to the downwelling region. Hence, there are downwelling flows at both the left and right sidewalls, and in the central part of the vessel. From 300 s, the pattern shows fluctuations and this continues for the duration of the experiment, suggesting the presence of oscillation of the organized flow structure, and the typical period is about $40 \mathrm{~s}$ for the case here. The \#1 and \#3 lines of measurement are at the same height in horizontally parallel positions as indicated in Fig. 1. Basically the fluctuating behavior is similar at these two lines, but there are differences of phase relating to the oscillation. Focusing on the time indices $\mathrm{t} 1$ to $\mathrm{t} 3$ in Fig. 2, the time lag of the flow pattern between the lines \#1 and \#3 is approximately half cycle which means the oscillatory deviation of the angle of roll axes, and there is also the short time lag between the waveforms on a single line which means the variation in the roll width. This feature is observed up to $1000 \mathrm{~s}$. The velocity of movement of the converging or diverging boundary is around $1 \mathrm{~mm} / \mathrm{s}$ at the position of the beam lines as read in the figure, and this velocity is lower than the observed horizontal flow velocity. Figure 3 shows an interpretation of the three-dimensional behavior of the organized flow for time $\mathrm{t} 1$ to $\mathrm{t} 3$, here there are four convection rolls as the mean flow with the oscillation of the roll axes, and each roll has also components of expansion and contraction.

In general, the flow pattern is strongly constrained by the geometry of the vessel, and the pattern in the present experiment has an oscillatory roll-like structure with the axis of 


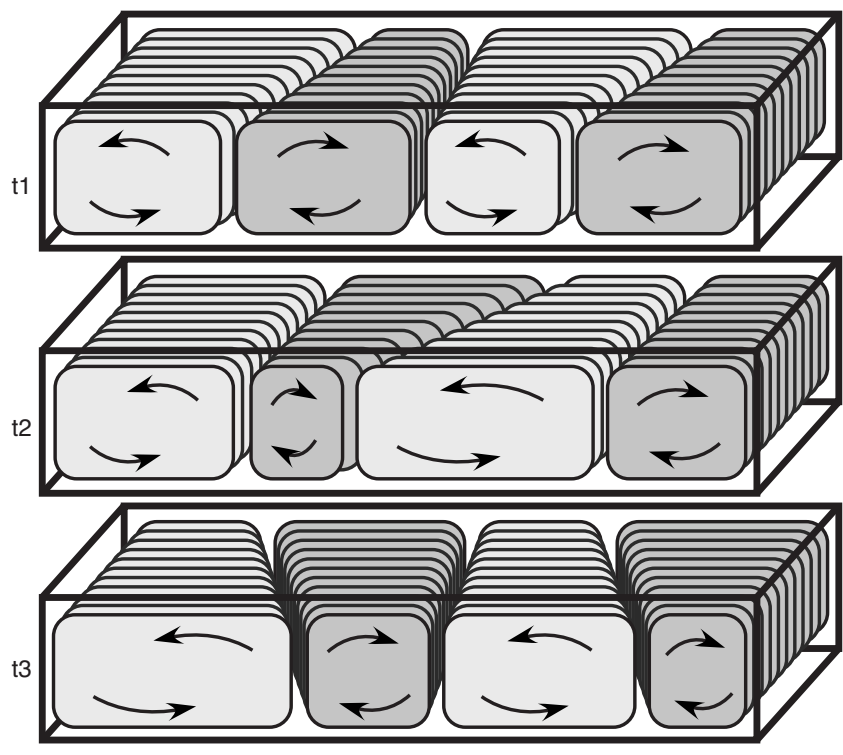

FIG. 3. Schematic representation of the three-dimensional organized flow structure and its oscillation inferred by the velocity measurements along two lines in Fig. 2. The time indices $\mathrm{t} 1$, $\mathrm{t} 2$, and $\mathrm{t} 3$ correspond to those in Fig. 2. Four convection rolls exist as a mean flow with periodic deviations of the roll axes, and each roll also displays components of expansion and contraction.

rotation perpendicular to the long horizontal length of the vessel. The number of rolls in the vessel depends on the initial conditions, e.g., the initial temperature distribution in the gallium, the small temperature differences between the vessel and gallium, and other parameters, as well as it also depends on the rapidity of the increase in the vertical temperature difference. In a different series of experiments, three cases with different numbers of rolls were observed, four, three, or two rolls, with the two-roll case least common. Overall it can be concluded that there is an oscillatory fluctuation in the roll structure. Once the number of rolls has stabilized in the process of formation of the large-scale flow, it lasts for the duration of an experiment. Both the number of rolls and the style of the fluctuations change little with increases or decreases of Rayleigh number, Ra for the $10^{4}-10^{5}$ range, though the typical velocity and fluctuation period depend on Ra. The following will analyze the case with a four rolls structure because it contains two central rolls that are not bounded by the sidewalls and allows a focus on the fluctuations in the flows.

\section{B. Simultaneous measurements of horizontal flow velocity and temperature}

To clarify the relation between the behavior of the flow and the temperature fluctuations, we made simultaneous measurements of the flow velocity and point measurements of the temperature. One line of measurement for UVP was set at position \#2 in Fig. 1, and three measurement points for the temperature by thermistor probes were set at the middle depth of the gallium layer, away from the ultrasonic beam line (ch1, ch2, and ch3 in Fig. 1).

Figure 4 shows the variations in the horizontal flow velocity and temperature during the simultaneous measure-

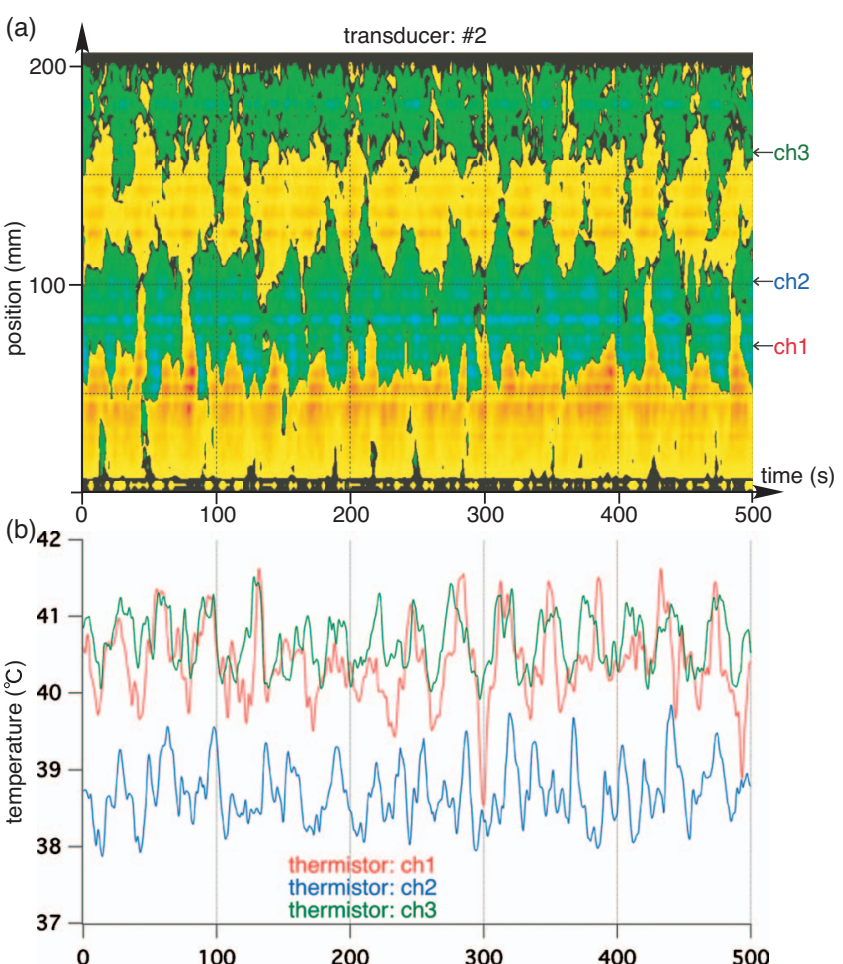

FIG. 4. (Color) Simultaneous measurements of horizontal flow velocities for position \#2 (a) and three points for the temperature (b) at $\mathrm{Ra}=1.4 \times 10^{5}$, the horizontal axis is the time. The color scale in the velocity graph is same as in Fig. 2. The positions of the termistor probes are indicated by $\operatorname{ch} 1$, ch2, and ch3. There is good correlation between the long-term oscillation $(\sim 30 \mathrm{~s})$ of the velocity profile and the temperature fluctuations, indicating that the fluctuation of the organized flow pattern is dominant in the fluctuation in the temperature.

ments for $500 \mathrm{~s}$. The convection is the thermally balanced state for $\mathrm{Ra}=1.4 \times 10^{5}$, and a four-roll pattern with oscillations is clearly observed. The spatiotemporal velocity distribution by the UVP [Fig. 4(a)] is similar to Fig. 2, showing that the flow structure in the vessel is similar to that in Fig. 3. Figure 4(b) displays the temperatures obtained by the three probes during these measurements. The velocity measurements indicate that the ch1 probe is located at the upwelling region of the second roll from the left sidewall, ch2 is at the downwelling region of the same second roll, and $\operatorname{ch} 3$ is at the upwelling region between the third and fourth rolls. The mean value of the temperature at ch2 is lower than at ch1 and $\operatorname{ch} 3$, as it is at the downwelling region of the large-scale flow. There is good correlation between the oscillation of the velocity profile and the temperature fluctuations at each of the probes in this figure. These results indicate that the movement of the upwelling and downwelling region of the rolllike structure induces the large amplitude of fluctuations in the temperature. There are 16 longer duration of large fluctuations for the $500 \mathrm{~s}$ both in velocity and temperature. Figure 5 displays the power spectrum density (PSD) for the velocity (a) and temperature (b) calculated from the time series in Fig. 4. The three velocity spectra correspond to the positions of the three thermistor probes. They are calculated from the spatially averaged value of the velocities at eight 

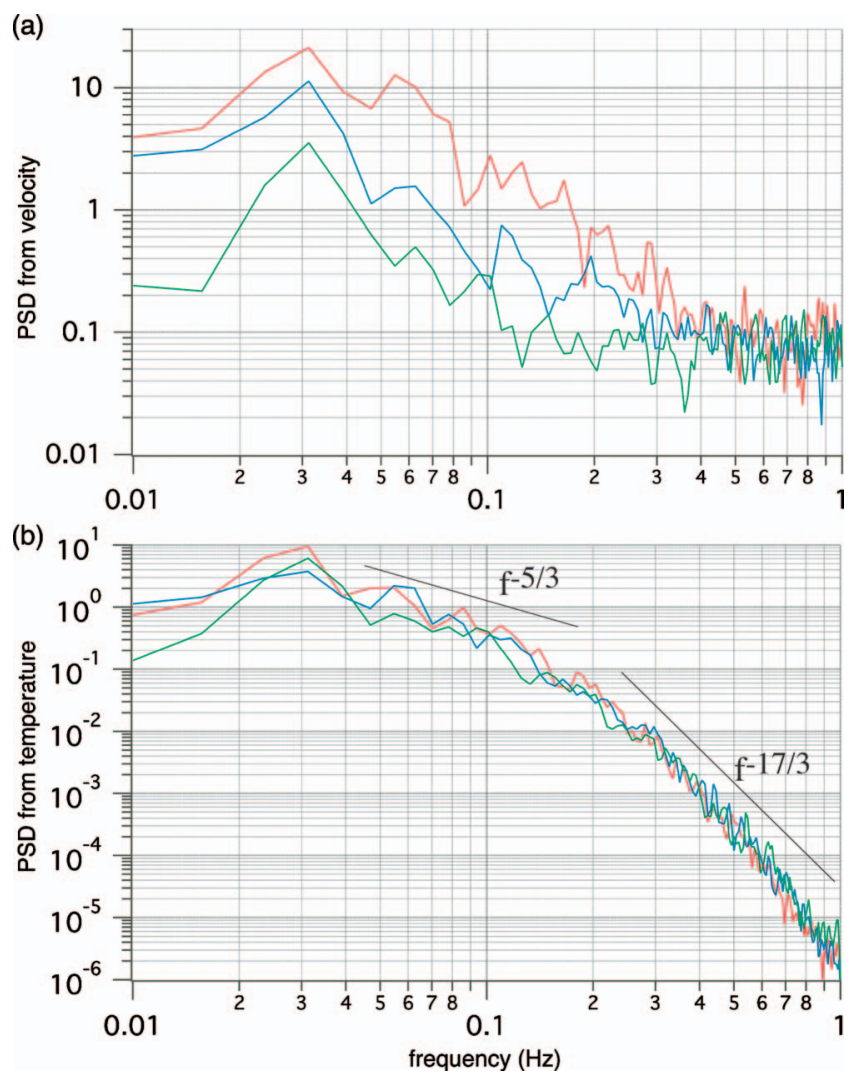

FIG. 5. (Color) (a) PSD graphs from the velocity time series around the positions of the thermistor probes: ch1 (red), ch2 (blue), and ch3 (green). (b) PSD graphs from the temperature time series for the three probes: ch1 (red), ch2 (blue), and ch3 (green). The peak frequency with the maximum power is common in the spectra, $0.03125 \mathrm{~Hz}$ (=32 seconds).

points $(\sim 10 \mathrm{~mm})$ centering on the position of the specific thermistor probe, as the velocity time series for individual points are very noisy. The peak frequency of the temperature measurements is in agreement with that of the velocity measurements, $0.03125 \mathrm{~Hz}$ (corresponding to the period of 32 s). Looking at the three PSDs for the temperature, the power at the peak frequency depends on the position of the probe related to the oscillating flow pattern, and ch1 shows the larger amplitude of fluctuations. Still, all these are roughly similar at the higher frequencies, and the decrease in power above $0.3 \mathrm{~Hz}$ lie on similar slopes. The value of the slope will be discussed in the next section. The peak power and the total power in the velocity spectrum is highest for the position around ch1 (red), and lowest for the position around ch3 (green). This is mainly due to the difference of the quality of velocity measurements between the positions, rather than reflecting the actual velocity fluctuations. In the farther half of the measurement line from the transducer, the data quality is lower due to the broadening of the ultrasonic beam and the scattering of the waves by the reflecting particles. The observed range of flow velocities in the present experiments is only 30 times the velocity resolution $(0.34 \mathrm{~mm} / \mathrm{s})$ with the noise level along the vertical axis around 0.1, making it difficult to discuss details of the rate of decrease in the power and details of the turbulence.

\section{Rayleigh number dependence of large-scale flow velocity and oscillation period}

A systematic investigation of the $\mathrm{Ra}$ dependence of the convective flow was also made. From the initial isothermal state, the temperature difference between the top and bottom plates was increased stepwise, maintaining the balanced state of the flow structure at each step, with simultaneous measurements of the flow velocity by the UVP (position \#2) and of the temperature by the three thermistor probes. The vertical temperature differences in the gallium layer were 0.6 , $1.1,2.1,3.7,6.9$, and $10.1 \mathrm{~K}$, corresponding to $\mathrm{Ra}$ values of $1.2 \times 10^{4}, 2.3 \times 10^{4}, 4.4 \times 10^{4}, 7.8 \times 10^{4}, 1.4 \times 10^{5}$, and $2.1 \times 10^{5}$, respectively. The critical $\mathrm{Ra}$ in this no-slip boundary condition is about 1700 for an infinite plane and slightly larger for this vessel, but is difficult to detect initiation of convection and emergence of oscillatory instability expected for just above the critical $\mathrm{Ra}$ with liquid metals, because of the very small velocities of the flows at Ra below $10^{4}$. The lowest $\mathrm{Ra}$ in the present measurement is $1.2 \times 10^{4}$, and it shows that a four-roll flow structure with oscillation is already established. The flow velocity and oscillation period clearly depend on Ra. The hysteresis of the flow behavior was checked by stepwise decreases in the temperature difference in the gallium layer, and it was confirmed that the pattern and statistical features are the same in both increasing and decreasing $\mathrm{Ra}$ between $4.4 \times 10^{4}$ and $2.1 \times 10^{5}$ so hysteresis does not exist in this range.

The following analyzes the six Ra values data set of the series of experiment with stepwise increases in Ra. The velocity measurements indicate that the basic flow pattern is four-roll structure and that it is preserved throughout the whole of the experiment. Figure 6 shows the time series of the temperature at the thermally balanced states for the six Ra values, for $500 \mathrm{~s}$ obtained by the ch1 probe. This probe is located at the upwelling region of the second convection roll from the left sidewall. The Ra of the system is increased by raising the temperature at the bottom, then the observed mean temperature is rising with $\mathrm{Ra}$, and the observed amplitude of the temperature fluctuations increases. For $\mathrm{Ra}=1.2$ $\times 10^{4}$, the waveform of the temperature fluctuations is simple sinusoidal and turbulence is not developed. This Ra is about five times higher than the boundary line for oscillatory instability in [5]. Above this line two-dimensional roll structure is unstable and theoretical study for the layer between horizontally infinite planes showed that traveling-wave convection exists and the wave propagates in the direction of roll axis [6]. In the present experiment, though the pattern is restricted by the sidewall, the observed structure for this $\mathrm{Ra}$ is consistent with the structure of oscillatory roll developed from the instability of the steady two-dimensional roll. The horizontal wave number for four-roll structure corresponds to $2.51(=\pi / 200 \mathrm{~mm} / 4 / 40 \mathrm{~mm})$ in the longer direction of the vessel, which is within the plausible range of wave number for the oscillatory roll [5]. These suggest that the emerged oscillatory instability as temperature difference between the top and bottom increases is basically preserved up to this Ra. The waveform of the temperature fluctuations becomes more complex with the increase in $\mathrm{Ra}$, indicating the development of turbulence. The PSDs of the temperature 

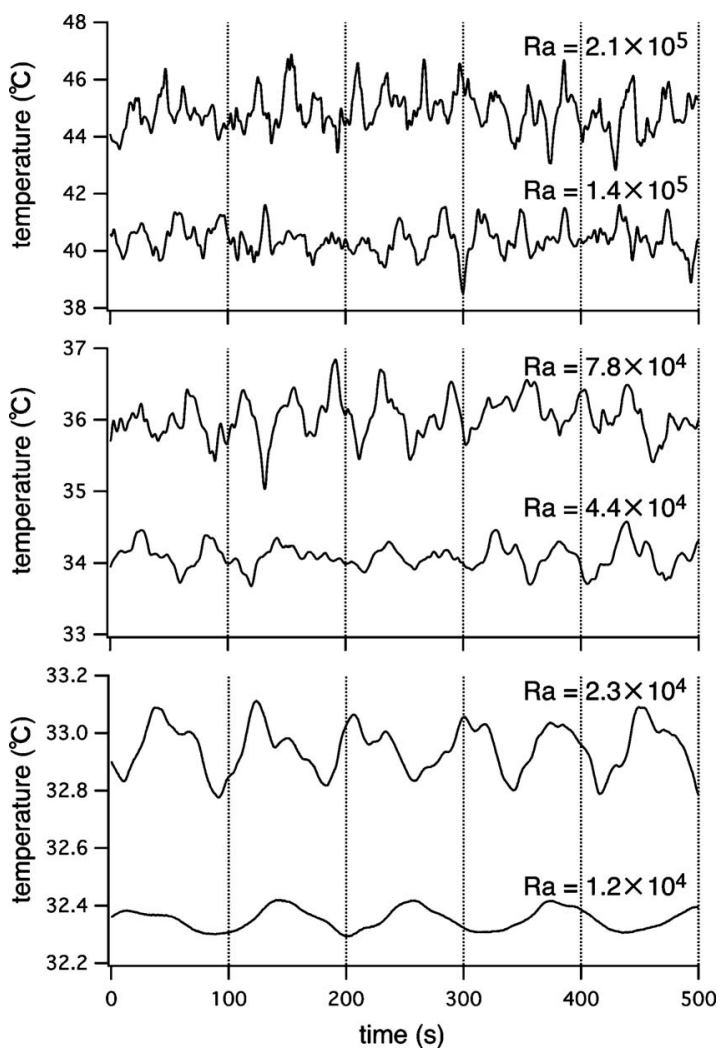

FIG. 6. Time series of temperature measurement over $500 \mathrm{~s}$ for six Rayleigh numbers. The data was obtained by the ch1 probe. From bottom to top: $\mathrm{Ra}=1.2 \times 10^{4}, 2.3 \times 10^{4}, 4.4 \times 10^{4}, 7.8 \times 10^{4}$, $1.4 \times 10^{5}$, and $2.1 \times 10^{5}$, respectively. The amplitude and frequency of the temperature fluctuations increase with increases in $\mathrm{Ra}$, at the same time the waveform becomes more complex.

calculated from these time series are shown in Fig. 7. They show that the peak frequency with the maximum power increases with the increase in Ra. The form of the PSDs depends on $\mathrm{Ra}$, and it shows a simple and rapid decrease of power for lower $\mathrm{Ra}\left(1.2 \times 10^{4}\right.$ and $\left.2.3 \times 10^{4}\right)$. The noise level $\left(\sim 10^{-6}\right)$ corresponds to the temperature resolution of the thermistor. From $\mathrm{Ra}=4.4 \times 10^{4}$, the rate of decrease in PSDs appears to change between the lower and higher frequency regions. This is clear for the two highest Ra, $1.4 \times 10^{5}$ and $2.1 \times 10^{5}$, where the PSDs decrease more gradually from the peak frequency to around $0.2 \mathrm{~Hz}$ than at the higher frequency region. The thermal energy spectrum model for low-Pr turbulence [19] predicts a $-5 / 3$ slope for the inertial-convective subrange and $\mathrm{a}-17 / 3$ slope for the inertial-conductive subrange. The slopes of PSDs for $\mathrm{Ra}=1.4 \times 10^{5}$ and $2.1 \times 10^{5}$ are consistent with this model, indicating the presence of an inertial-convective subrange at $0.05 \sim 0.2 \mathrm{~Hz}$ and that the inertial-conductive subrange corresponds to higher than 0.3 $\mathrm{Hz}$ at $\mathrm{Ra}=2.1 \times 10^{5}$. These two slopes are indicated in the figure. The transformation of the temperature PSDs in the present experiment suggests that the flow becomes highly turbulent on $\mathrm{Ra} \sim 10^{5}$, and the velocity measurements made clear that the basic flow pattern identified as large-scale flow in liquid metal has a continuity from the oscillatory convection emerged at low Ra. Experiments with liquid sodium [7] observed $\mathrm{a}-4$ slope for $\mathrm{Ra} \sim 10^{6}$ and noted that there is

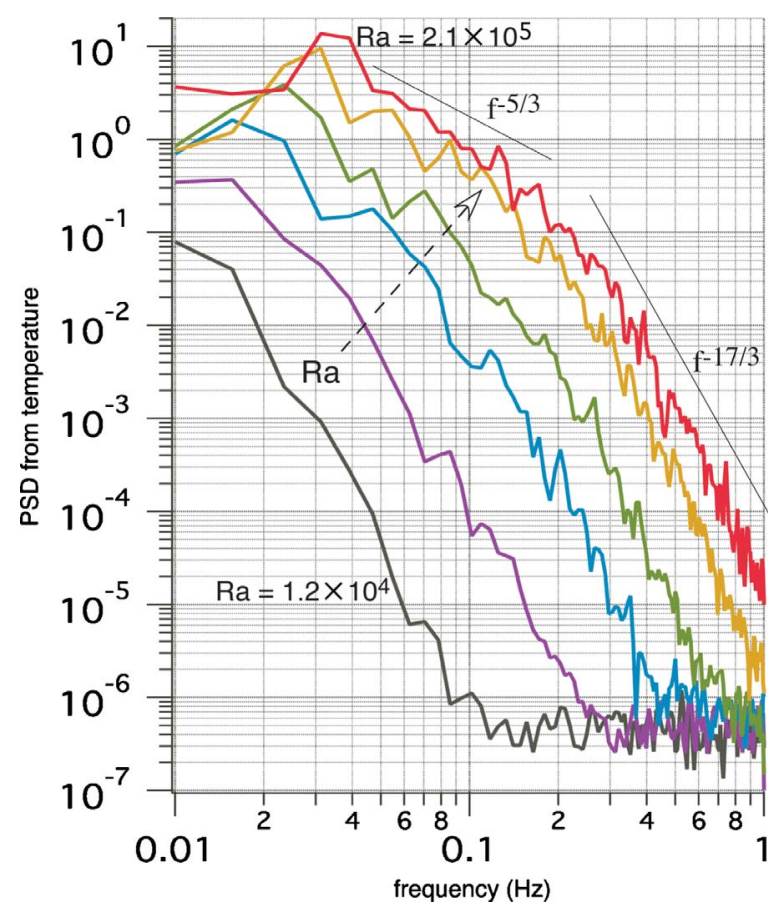

FIG. 7. (Color online) PSD graphs from the time series of the temperature shown in Fig. 6. The peak frequency and its amplitude increase with increases in $\mathrm{Ra}$. The slopes predicted by the spectral model for low-Pr turbulence, $-5 / 3$ and $-17 / 3$, are indicated in the figure. The form of the PSD graphs for $\mathrm{Ra}=1.4 \times 10^{5}$ and 2.1 $\times 10^{5}$ are consistent with the spectral model.

neither an internal-convective subrange nor an inertialconductive subrange in the flow. The results here suggest that the differences could arise from the different geometries of the vessels. In [7] the vessel was a cylindrical cell with large aspect ratio, and the basic flow pattern was concentric ring rolls.

With increases in Ra, the UVP measurement shows that the observed velocity of the flow becomes higher, and the typical horizontal flow velocity $U_{H}$ is here defined as the maximum velocity observed in a cycle of oscillation for the second roll from the left sidewall. This criterion is introduced as the quality of the velocity measurements depend on the distance from the transducer, and the region must be similar for quantitative comparisons of the velocities. With this criterion, $U_{H}$ is $4.5 \mathrm{~mm} / \mathrm{s}$ for the case of the velocity measurements shown in Fig. 4. Figure 8 displays the Ra dependence of the velocity in nondimensional form, $U_{H} L / \kappa$, with $L$ the layer thickness $(40 \mathrm{~mm})$ and $\kappa$ the thermal diffusivity of liquid gallium $\left(1.18 \times 10^{-5} \mathrm{~m}^{2} / \mathrm{s}\right)$. The error bar is from the range of the maximum velocities observed for all oscillations, and it is relatively larger for lower Ra values because of the limitations of the velocity resolution. This graph shows that $U_{H}$ is approximately proportional to the square root of $\mathrm{Ra}$, though the range of the exponents is large $(0.51 \pm 0.14)$. The fitted line in the graph is $U_{H} L / \kappa=0.05$ $\times \mathrm{Ra}^{0.51}$. The $\mathrm{Ra}^{1 / 2}$ dependence of the typical velocity from these measurements is consistent with theoretical predictions for low Pr fluids by [16]. The $\mathrm{Ra}^{3 / 7}$ dependence of the velocity is proposed for hard turbulence in [20], but the present 


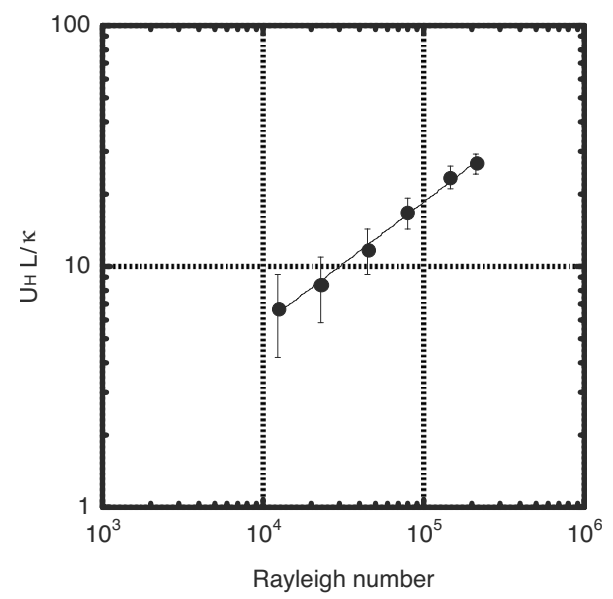

FIG. 8. The relation between Rayleigh number and nondimensional velocity $U_{H} L / \kappa . U_{H}$ is the typical horizontal velocity of the convective flow; it is nondimensionalized by the layer depth $L$, and the thermal diffusivity of gallium $\kappa$. The fitting line is $U_{H} L / \kappa=0.05 \times \mathrm{Ra}^{0.51}$.

data are not sufficiently precise to distinguish the $1 / 2$ and $3 / 7$ exponents.

The oscillating patterns of the gallium convection were observed by UVP measurements for the six Ra values in this series discussed here, and the following compares the typical frequency of oscillation observed by the temperature and velocity measurements. The typical frequency $f_{p}$ is determined by the peak frequency with maximum power in the specific PSD, but the velocity data sets for $\mathrm{Ra}=1.2 \times 10^{4}$ and 2.3 $\times 10^{4}$ have very weak signals to determine the peak frequency and instead the number of oscillations from the velocity profile for $500 \mathrm{~s}$ was counted. These estimates of the typical frequency are shown in nondimensional form $f_{p} L^{2} / \kappa$ in Fig. 9. The error is estimated by the width of the peak or the uncertainty in the oscillation count. This graph shows that the typical frequencies are very similar for both the tem-

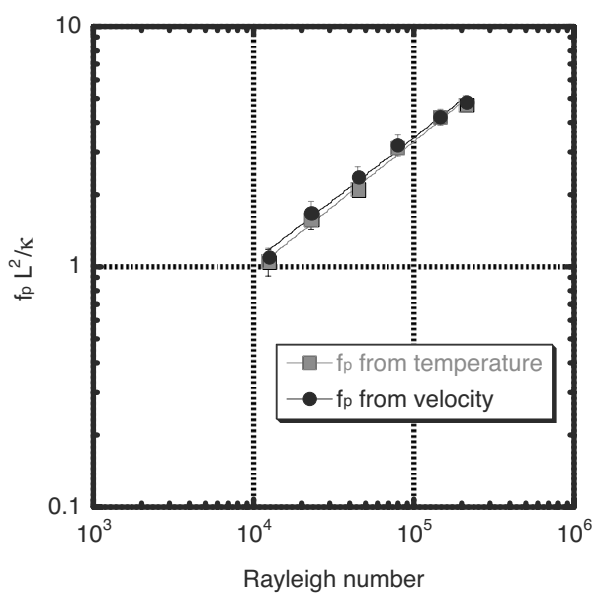

FIG. 9. The relation between Rayleigh number and nondimensional frequency $f_{p} L^{2} / \kappa . f_{p}$ is the peak frequency of the power spectrum of temperature or velocity. The two frequencies agree well at all $\mathrm{Ra}$ values. The fitting line from the temperature data set is $f_{p} L^{2} / \kappa=0.007 \times \mathrm{Ra}^{0.53}$, and that from the velocity data set is $f_{p} L^{2} / \kappa=0.009 \times \mathrm{Ra}^{0.51}$.

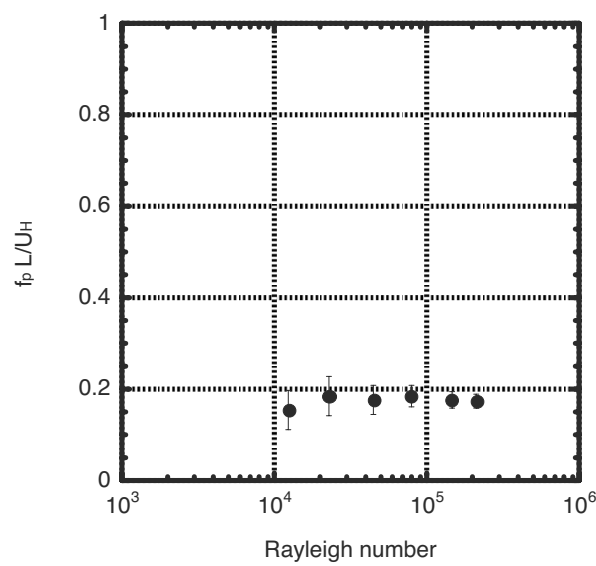

FIG. 10. The relation between Rayleigh number and nondimensional ratio of frequency to velocity $f_{p} L / U_{H}$. The ratio does not depend on $\mathrm{Ra}$, and is a constant value of about 0.18 .

perature and velocity measurements, and $f_{p}$ is approximately proportional to the square root of Ra. The estimated range of the exponent is $0.52 \pm 0.05$. The lines in the graph are $f_{p} L^{2} / \kappa=0.007 \times \mathrm{Ra}^{0.53}$ from the temperature measurements, and $f_{p} L^{2} / \kappa=0.009 \times \mathrm{Ra}^{0.51}$ from the velocity measurements. The $\mathrm{Ra}^{1 / 2}$ dependence of the frequency is consistent with the theoretical prediction for oscillatory convection in low-Pr fluid by [4]. Moreover, this is in agreement with the reported dependence of frequency on Ra by temperature measurements in mercury with small aspect ratio cylinder for Ra $\geq 10^{6}[10]$, [11]. In their cases, the flow is extremely turbulent and whole cylinder-scale flow is supposed to exist. The present result for moderate Ra can connect between low and high $\mathrm{Ra}$ ranges with the same frequency scaling.

The results here establish the relation between the flow velocity and oscillation period of a pattern for the liquid gallium convection in this vessel. In the range of Ra values of the present experiments, both $U_{H}$ and $f_{p}$ are approximately proportional to $\mathrm{Ra}^{1 / 2}$, hence the $f_{p}$ to $U_{H}$ ratio does not depend on $\mathrm{Ra}$. The nondimensional value of $f_{p} L / U_{H}$ is shown in Fig. 10. Here the value of $f_{p}$ from the velocity fluctuations was used. This graph suggests that $f_{p} L / U_{H}$ is constant and its value about 0.18 . This result, that $f_{p} L / U_{H}$ has a constant value, is consistent with the velocity measurements in the large-scale flow of mercury for $\mathrm{Ra} \geq 10^{6}$ [15]. The $f_{p} L / U_{H}=0.18$ (that is, $1 / f_{p}=5.6 L / U_{H}$ ) relation can be understood by assuming the conservation of mass between the horizontal and vertical flows, then the typical vertical flow velocity is inversely proportional to the aspect ratio of the convection roll. In the present experiment the aspect ratio of a roll is $5 / 4$, and the assumed vertical velocity is $4 / 5 U_{H}$. Then the time of circulation for the flow in a roll is 2 $\times\left(\frac{5 / 4 L}{U_{H}}+\frac{L}{4 / 5 U_{H}}\right)=\frac{5 L}{U_{H}}$, which is comparable to the observed typical period, $1 / f_{p}$. This is the standard interpretation of the peak frequency for an aspect ratio $\sim 1$ vessel [11], [15]. An alternative interpretation would be to consider the horizontal scale of the vessel. The horizontal length of this vessel is $5 L$, giving a crossing time for the horizontal flow $U_{H}$ as $5 \mathrm{~L} / U_{H}$, which is comparable to the typical period of the oscillation, $1 / f_{p}$. The validity of these two interpretations may be evaluated by comparing the situation with different numbers of 
rolls. It is desirable to make measurements with wider aspect ratio vessels, because when the number of roll is low, the roll is bounded by the sidewalls and its freedom of oscillation is restricted.

\section{CONCLUDING REMARKS}

The results here report the simultaneous measurement of velocity profile and temperature for Rayleigh-Bénard convection at $\mathrm{Pr}=0.025$ in the Rayleigh number range from 1 $\times 10^{4}$ to $2 \times 10^{5}$. The measurement of the horizontal flow velocity at two lines in the vessel expresses the threedimensional structure of the roll-like pattern, coexisting with oscillatory fluctuations. From $\mathrm{Ra} \sim 10^{5}$, temperature PSDs show the typical feature of developed turbulence expected for low Pr fluid in high frequency, and the roll-like pattern observed in these $\mathrm{Ra}$ is a large-scale flow that is the organized structure in the turbulence. The basic structure of the large-scale flow at this $\mathrm{Ra}$ can be thought continuously developed from the oscillatory instability of the twodimensional roll emerged just above the critical Ra. The fluc- tuation of the large-scale flow is characterized by the oscillation of the roll axes, and each roll also has the components of expansion and contraction. The horizontal motion of the roll induces the long-term fluctuation of temperature with large amplitude as indicated by the simultaneous measurements of velocity and temperature. The Rayleigh number dependence of the flow features for a convection pattern with four roll was analyzed, and the typical horizontal flow velocity increases proportional to $\mathrm{Ra}^{1 / 2}$ in the range $1 \times 10^{4} \leq \mathrm{Ra}$ $\leq 2 \times 10^{5}$. The dependence of the typical frequency of the roll oscillation is also $\mathrm{Ra}^{1 / 2}$, which suggests a close relation between the emergence mechanism and behavior of the large-scale flow in highly turbulent state and the oscillatory instability of the two-dimensional roll structure in the low-Ra region.

\section{ACKNOWLEDGMENT}

This work was supported by the Grants-in-Aid for Scientific Research No. 18204038 from the Ministry of Education, Culture, Sports, Science and Technology, Japan. The authors express thanks for this support.
[1] R. Krishnamurti, J. Fluid Mech. 42, 295 (1970).

[2] R. Krishnamurti and L. N. Howard, Proc. Natl. Acad. Sci. U.S.A. 78, 1981 (1981).

[3] G. Ahlers, S. Grossmann, and D. Lohse, Rev. Mod. Phys. 81, 503 (2009).

[4] F. H. Busse, J. Fluid Mech. 52, 97 (1972).

[5] R. M. Clever and F. H. Busse, J. Fluid Mech. 65, 625 (1974).

[6] R. M. Clever and F. H. Busse, J. Fluid Mech. 176, 403 (1987).

[7] S. Horanyi, L. Krebs, and U. Müller, Int. J. Heat Mass Transfer 42, 3983 (1999).

[8] U. Burr and U. Müller, Phys. Fluids 13, 3247 (2001).

[9] J. M. Aurnou and P. L. Olson, J. Fluid Mech. 430, 283 (2001).

[10] T. Takeshita, T. Segawa, J. A. Glazier, and M. Sano, Phys. Rev. Lett. 76, 1465 (1996).

[11] S. Cioni, S. Ciliberto, and J. Sommeria, J. Fluid Mech. 335, 111 (1997).

[12] T. Segawa, A. Naert, and M. Sano, Phys. Rev. E 57, 557
(1998).

[13] Y. Takeda, JSME Int. J., Ser. B 38, 8 (1995).

[14] T. Mashiko, Y. Tsuji, T. Mizuno, and M. Sano, Phys. Rev. E 69, 036306 (2004).

[15] Y. Tsuji, T. Mizuno, T. Mashiko, and M. Sano, Phys. Rev. Lett. 94, 034501 (2005).

[16] F. H. Busse and R. M. Clever, J. Fluid Mech. 102, 75 (1981).

[17] Y. Tasaka, Y. Takeda, and T. Yanagisawa, Flow Meas. Instrum. 19, 131 (2008).

[18] D. Brito, H. C. Nataf, J. Aubert, and J. P. Masson, Exp. Fluids 31, 653 (2001).

[19] G. K. Batchelor, I. D. Howells, and A. A. Townsend, J. Fluid Mech. 5, 134 (1959).

[20] B. Castaing, G. Gunaratne, F. Heslot, L. Kadanoff, A. Libchaber, S. Thomae, X. Z. Wu, S. Zaleski, and G. Zanetti, J. Fluid Mech. 204, 1 (1989). 